\title{
Cyclic branched coverings and homology 3-spheres with large group actions
}

\author{
by \\ Bruno P. Zimmermann (Trieste)
}

\begin{abstract}
We show that, if the covering involution of a 3-manifold $M$ occurring as the 2 -fold branched covering of a knot in the 3 -sphere is contained in a finite nonabelian simple group $G$ of diffeomorphisms of $M$, then $M$ is a homology 3-sphere and $G$ isomorphic to the alternating or dodecahedral group $\mathbb{A}_{5} \cong \operatorname{PSL}(2,5)$. An example of such a 3 -manifold is the spherical Poincaré sphere. We construct hyperbolic analogues of the Poincaré sphere. We also give examples of hyperbolic $\mathbb{Z}_{2}$-homology 3 -spheres with $\operatorname{PSL}(2, q)$-actions, for various small prime powers $q$. We note that the groups $\operatorname{PSL}(2, q)$, for odd prime powers $q$, are the only candidates for being finite nonabelian simple groups which possibly admit actions on $\mathbb{Z}_{2}$-homology 3 -spheres (but the exact classification remains open).
\end{abstract}

1. Introduction. By [Z1], the only finite nonabelian simple group acting on a homology 3 -sphere is the alternating or dodecahedral group $\mathbb{A}_{5} \cong$ $\operatorname{PSL}(2,5)$. Up to conjugation, there are two orthogonal actions of $\mathbb{A}_{5}$ on the 3 -sphere, in each case the quotient orbifold is the 3 -sphere, and the singular sets of the two actions are the graphs shown in Figures $1 \mathrm{a}$ and $\mathrm{b}$ (where edges are labelled with their branching orders, and edges without label have branching order two). Apart from this, the most prominent example of a homology 3 -sphere with an $\mathbb{A}_{5}$-action is the Poincaré sphere; again the quotient orbifold is the 3 -sphere, and the singular set the tetrahedral graph shown in Figure 1c. The Poincaré sphere is a spherical manifold, and the group $\mathbb{A}_{5}$ acts by isometries. The cyclic subgroups of orders two, three and five of $\mathbb{A}_{5}$, unique up to conjugation, are the covering groups of the Poincaré sphere as the 2 -fold branched covering of the $(3,5)$-torus knot, the 3 -fold cyclic branched covering of the $(2,5)$-torus knot and the 5 -fold cyclic branched covering of the $(2,3)$-torus knot; on the other hand, the isometry group of the Poincare sphere is isomorphic to the orthogonal group $S O(3)$, and the

2000 Mathematics Subject Classification: 57M12, 57M25, 57M50, 57M60, 57S17.

Key words and phrases: homology 3-sphere, hyperbolic 3-manifold, cyclic branched covering, simple group action, dodecahedral group. 
three covering groups are conjugate in the isometry group of the Poincaré sphere to subgroups of a single cyclic group of order 30, and consequently these three knots are related by the second standard abelian construction described in [RZ1]. We note that, in general, it is quite a rare phenomenon that the same 3-manifold occurs as a cyclic branched covering of different knots in $S^{3}$ (see [RZ1], [RZ2]).

Our first result is the following

THEOREM 1. Let $M$ be the 2-fold branched covering of a knot in the 3sphere such that the covering involution is an element of a finite nonabelian simple group $G$ of diffeomorphisms of $M$. Then $M$ is a homology 3-sphere, and $G$ is isomorphic to the dodecahedral group $\mathbb{A}_{5}$.

We believe that Theorem 1 also remains true for $p$-fold cyclic branched coverings, for primes $p>2$.

The Poincaré sphere is the only spherical homology 3-sphere (and, as part of the 3-manifold geometrization program, conjectured to be the only homology 3 -sphere with finite fundamental group). There are no euclidean homology 3-spheres (in fact, not a single example of a closed euclidean homology sphere seems to be known in any dimension). There are many hyperbolic homology 3-spheres, and the question arises if there exists one with properties similar to the Poincaré sphere. We have the following

TheOREM 2. There exists a hyperbolic homology 3-sphere $M$ (of approximate volume 32.01607 ) which is the 2 -fold branched covering of a knot $K_{2}$ in $S^{3}$ and the 3-fold cyclic branched covering of a knot $K_{3}$ in $S^{3}$, such that the covering groups generate a group $\mathbb{A}_{5}$ of isometries of $M$. The quotient orbifold $M / \mathbb{A}_{5}$ is the 3 -sphere, and its singular set is the Kuratowski graph shown in Figure 1d (the complete bi-partite graph on six vertices).

In the language of [RZ3] this means that the hyperbolic 3-manifold $M$ has many hidden symmetries with respect to both the 2 -fold and the 3 -fold cyclic branched coverings (i.e., symmetries which are not lifts of symmetries of the knots $K_{2}$ resp. $K_{3}$; this is again a quite exceptional situation). The manifold $M$ is also the 5 -fold cyclic branched covering of a knot $K_{5}$ in a homology 3 -sphere different from $S^{3}$; at present we do not have an example of a hyperbolic homology 3 -sphere with an $\mathbb{A}_{5}$-action such that all three knots $K_{2}, K_{3}$ and $K_{5}$ are knots in $S^{3}$ (as in the case of the Poincaré sphere).

We note that the covering groups of $K_{2}$ and $K_{3}$ in Theorem 2 can be chosen to lie in a dihedral subgroup $\mathbb{D}_{3}$ of order six of $\mathbb{A}_{5}$, and that consequently we are in the standard dihedral situation described in [RZ2] for two knots with the same 2-fold resp. 3-fold cyclic branched covering. 
In Section 5, we describe other hyperbolic homology 3-spheres with an isometric $\mathbb{A}_{5}$-action which occur as cyclic branched coverings of knots in the 3 -sphere; one of these is a 3 -fold and also a 5 -fold cyclic branched covering of knots in the 3 -sphere. This seems to be the first example of two knots in the 3 -sphere which have the same hyperbolic 3-manifold as a cyclic branched covering but which are not related in a solvable way (i.e., the covering groups cannot be chosen to generate a solvable group of isometries of the manifold; see also $[$ RZ1]).

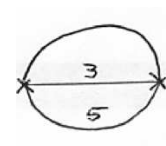

a)

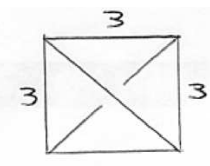

b)

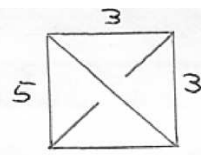

c)

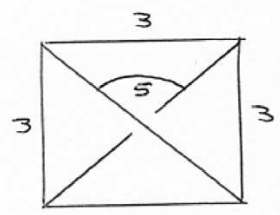

d)

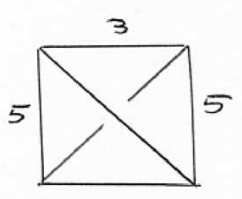

e)

Fig. 1

The best known hyperbolic 3 -manifold with an isometric $\mathbb{A}_{5}$-action is the Seifert-Weber dodecahedral manifold. The singular set of the quotient orbifold by the $\mathbb{A}_{5}$-action is the tetrahedral graph shown in Figure 1e; the fact that the edges of order five are disconnected implies that the SeifertWeber manifold is not a $\mathbb{Z}_{5}$-homology 3 -sphere (i.e., with the homology of the 3-sphere with coefficients in the integers mod five; in fact, by $[\mathrm{Br}$, Theorem 7.9] the fixed point set of a periodic transformation of order five of a $\mathbb{Z}_{5}$-homology 3 -sphere is connected). The Seifert-Weber manifold is a 5 -fold cyclic branched covering of the 2-component Whitehead link (which again implies that it is not a $\mathbb{Z}_{5}$-homology 3 -sphere; in fact, its first homology is $\left.\left(\mathbb{Z}_{5}\right)^{3}\right)$; it is also the 2-fold and 3-fold cyclic branched covering of knots $K_{2}$ resp. $K_{3}$ in lens spaces with fundamental group $\mathbb{Z}_{5}$.

Considering the more general class of $\mathbb{Z}_{2}$-homology 3 -spheres, it is shown in [MZ] that the only finite simple groups which possibly admit actions on $\mathbb{Z}_{2}$-homology 3 -spheres are the linear fractional groups $\operatorname{PSL}(2, q)$, for odd prime powers $q$; however, it remains open for which odd prime powers $q$ such actions really occur. In Section 4 , we give examples of $\operatorname{PSL}(2, q)$-actions on $\mathbb{Z}_{2}$-homology 3 -spheres, for various small values of $q$. These examples are 
obtained as regular branched coverings of suitable 3-orbifolds, and we calculate the homology of these coverings by computer using the group-theory package GAP (we thank M. Conder for his help with these examples). We think that all such groups $\operatorname{PSL}(2, q)$ admit actions on $\mathbb{Z}_{2}$-homology 3 -spheres but have no method to prove this at present.

2. Proof of Theorem 1. It is well known that, for any prime power $p^{n}$, the $p^{n}$-fold cyclic branched covering of a knot in $S^{3}$ is a $\mathbb{Z}_{p}$-homology 3sphere (see e.g. [G]), so $M$ is a $\mathbb{Z}_{2}$-homology 3 -sphere. It follows from [MZ, Theorem 1] that a finite simple group $G$ which admits an action on a $\mathbb{Z}_{2^{-}}$ homology 3 -sphere is isomorphic to a linear fractional groups $\operatorname{PSL}(2, q)$, for an odd prime power $q$. Alternatively, one may apply [RZ4], where it is shown that a finite simple group $G$ acting on a closed 3-manifold and containing an involution $h$ with nonempty connected fixed point set is isomorphic to a group $\operatorname{PSL}(2, q)$, for an odd prime power $q$.

So we can assume that $G=\operatorname{PSL}(2, q)$, where $q$ is an odd prime power. In $G=\operatorname{PSL}(2, q)$, all involutions are conjugate, and in particular conjugate to the covering involution $h$. Then the quotient of $M$ by any involution in $G$ is the 3 -sphere with a knot as branch set.

A Sylow 2-subgroup of $G=\operatorname{PSL}(2, q)$ is a dihedral group which contains a subgroup $U \cong \mathbb{Z}_{2} \times \mathbb{Z}_{2}$. The quotient of $M$ by each of the three involutions $h_{1}, h_{2}$ and $h_{3}$ in $U$ is the 3 -sphere. The group $U$ projects to an involution $\bar{h}$ of $M / h_{1} \cong S^{3}$, with nonempty fixed point set. By the solution of the Smith conjecture for involutions ([W]), the involution $\bar{h}$ of $S^{3}$ is standard (conjugate to an orthogonal one), and hence $S^{3} / \bar{h}=M / U$ is again the 3-sphere. So the space of the quotient orbifold $M / U$ is $S^{3}$, and its singular set is a $\theta$-curve $T$ in $S^{3}$ (i.e. a graph with two vertices and three connecting edges), with branching order two associated to the three edges (because $h_{2}$ and $h_{3}$ act as strong inversions on the fixed point set of $h_{1}$ ). The three edges of such a $\theta$-curve define three knots and hence three 2-fold branched coverings of $S^{3}$ along these knots. In our situation, these three 2-fold branched coverings are the 3-manifolds $M / h_{1}, M / h_{2}$ and $M / h_{3}$, all homeomorphic to the 3 -sphere. Also, $M$ is the regular $\mathbb{Z}_{2} \times \mathbb{Z}_{2}$-covering of $S^{3}$ branched along the $\theta$-curve $T$.

By [N1] or [N2], the first homology of the regular $\mathbb{Z}_{2} \times \mathbb{Z}_{2}$-covering of a $\theta$-curve in the 3 -sphere is the product of the first homologies of the three 3 -manifolds which are the 2 -fold branched coverings associated to the three constituent knots of the $\theta$-curve. In our case, we get three times the 3 sphere, which implies that $M$ is an integer homology 3-sphere. By [Z1], the only finite simple group acting on an integer homology 3 -sphere is the alternating or dodecahedral group $\mathbb{A}_{5} \cong \operatorname{PSL}(2,5)$.

This finishes the proof of Theorem 1 . 
3. Proof of Theorem 2. We denote by $\mathcal{K}$ the 3 -orbifold whose space is the 3 -sphere and whose singular set is the Kuratowski graph shown in Figure 1d resp. 2a. Using the orbifold geometrization theorem ([BP], [CHK]) we will show that $\mathcal{K}$ is a hyperbolic orbifold (alternatively, one may try to construct explicitly its universal covering group in hyperbolic 3 -space).

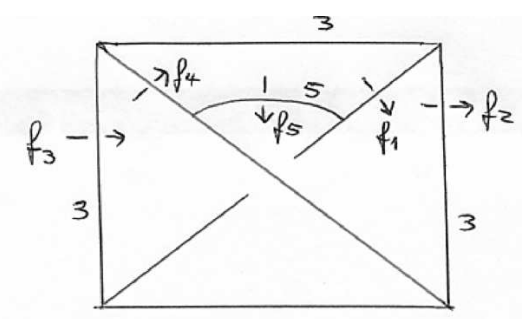

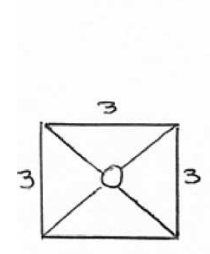

b)

\section{a)}

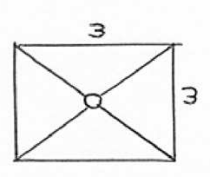

c)

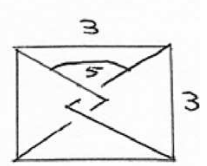

d)

Fig. 2

We consider the spherical 3 -orbifold $\mathcal{O}$ whose space is the 3 -sphere and whose singular set is the graph shown in Figure 1b. Let $p: S^{3} \rightarrow \mathcal{O}$ be its univeral covering, with covering group $\mathbb{A}_{5}$ acting orthogonally on the 3 -sphere. Note that the singular graph of the orbifold $\mathcal{K}$ is obtained by adjoining the unique edge $\bar{L}$ of branching order five to the singular graph of $\mathcal{O}$ (see Figures $1 \mathrm{~d}$ and $1 \mathrm{~b}$ ). The complement of a regular neighbourhood of the edge $\bar{L}$ in $\mathcal{K}$ is the pyramidal orbifold $\widetilde{\mathcal{P}}$ with one cusp whose singular set is shown in Figure 2b. Now $\widetilde{\mathcal{P}}$ is a complete hyperbolic 3-orbifold of finite volume; in fact, $\widetilde{\mathcal{P}}$ is a 2 -fold (branched) covering of the Picard orbifold $\mathcal{P}$ shown in Figure 2c, i.e. the quotient of hyperbolic 3-space by the Picard group $\operatorname{PSL}(2, \mathbb{Z}[i])$ (see e.g. $[\mathrm{F}]$ ).

Let $L$ be the link in $S^{3}$ which is the preimage of the edge $\bar{L}$ under the covering $p: S^{3} \rightarrow \mathcal{O}$; the complement of $L$ is hyperbolic since it is a finite cover of the hyperbolic orbifolds $\widetilde{\mathcal{P}}$ and $\mathcal{P}$, so $L$ is a hyperbolic link invariant under the $\mathbb{A}_{5}$-action on $S^{3}$ (at present we do not have an explicit projection of this "dodecahedral link" $L$; the volume of its complement is 36.63852 , which is 120 times the volume 0.305321 of the Picard orbifold). Let $\mathcal{L}_{5}$ be the closed 3 -orbifold with singular set $L$ obtained by associating branching order five to all components of the link $L$; by the orbifold geometrization 
theorem, and in particular [BP, Corollary 3], $\mathcal{L}_{5}$ is a hyperbolic orbifold. By a second application of the orbifold geometrization theorem we can suppose that the group $\mathbb{A}_{5}$ of symmetries of the link $L$ acts by hyperbolic isometries of the orbifold $\mathcal{L}_{5}$. The quotient of $\mathcal{L}_{5}$ by the $\mathbb{A}_{5}$-action is the orbifold $\mathcal{K}$, so also $\mathcal{K}$ is a hyperbolic orbifold (at the end of this section, we will compute the volume of $\mathcal{K}$ ).

We will calculate a presentation of the orbifold fundamental group $\pi_{1} \mathcal{K}$ of $\mathcal{K}$. The fundamental group of the complement in $S^{3}$ of the singularity graph of $\mathcal{K}$ can be computed from its projection in Figure 2a by the Wirtinger algorithm, in a similar way to the fundamental group of the complement of a knot in $S^{3}$, calculated from a projection of the knot. The fundamental group of the complement is generated by the meridians of all edges of $\mathcal{K}$; in Figure 2a, we have indicated some of these meridian generators. For each vertex and each crossing of the singular set we obtain a relation (as in the case of knots or links one of these relations is redundant). Then a presentation of the orbifold fundamental group $K:=\pi_{1} \mathcal{K}$ is obtained by raising all meridian generators to the powers given by the branching orders. Using the relations to eliminate some of the meridian generators, we obtain the following presentation of $K$ :

$$
\begin{aligned}
\left\langle f_{1}, f_{2}, f_{3}, f_{4}, f_{5}\right| f_{1}^{2}, f_{2}^{3}, f_{3}^{3}, f_{4}^{2}, f_{5}^{5},\left(f_{1} f_{2}\right)^{3}, f_{3} f_{4} f_{1} f_{2}, & \left.\left(f_{4} f_{5}\right)^{2},\left(f_{1} f_{5}\right)^{2},\left(f_{4} f_{5} f_{2}\right)^{2}\right\rangle .
\end{aligned}
$$

We define a surjection $\phi: K \rightarrow \mathbb{A}_{5}$ by

$$
\begin{gathered}
\phi\left(f_{1}\right)=(1,4)(2,3), \quad \phi\left(f_{2}\right)=(2,4,3), \quad \phi\left(f_{3}\right)=(2,3,4), \\
\phi\left(f_{4}\right)=(1,4)(2,3), \quad \phi\left(f_{5}\right)=(1,2,3,4,5) .
\end{gathered}
$$

The kernel $U$ of $\phi$ is torsionfree (the torsion elements of $K$ are conjugate to the meridians of the singular edges of $\mathcal{K}$ ). Now $M:=\mathbb{H}^{3} / U$ is a closed hyperbolic 3-manifold, with fundamental group isomorphic to $U$, and with an induced action of $\mathbb{A}_{5} \cong K / U$ such that $M / \mathbb{A}_{5}=\mathbb{H}^{3} / K=\mathcal{K}$. Using computational techniques, and in particular the group theory package GAP, we find that the abelianization of $U \cong \pi_{1} M$ is trivial, which implies that $M$ is an integer homology 3 -sphere.

We consider the involution $h=\phi\left(f_{1}\right)$ in $\mathbb{A}_{5}$ (note that all involutions in $\mathbb{A}_{5}$ are conjugate). The fixed point set of $h$ is nonempty (because $\mathcal{K}$ has singular edges of order two), and by [Br, Theorem 7.9] the fixed point set of $h$ is connected (because $M$ is a homology 3 -sphere). Then $M$ is a 2 -fold branched covering of the 3-manifold $M_{2}:=M / h$, branched along a knot $K_{2}$ in $M_{2}$. We denote by $U_{2}$ the subgroup of $K$ generated by $U$ and the element $f_{1}$ of order two; then $U$ is a subgroup of index two of $U_{2}$, and $M_{2}=M / h=\mathbb{H}^{3} / U_{2}$. By [A], the fundamental group of $M_{2}$ is obtained by 
dividing out the torsion of $U_{2}$, that is, by taking the quotient of $U_{2}$ by the normal subgroup generated by $f_{1}$.

GAP produces a presentation of the kernel $U$ of $\phi$ with 10 generators (given as words in the generators $f_{i}$ of $K$ ) and 16 relations. We add $f_{1}$ to these generators and obtain a generating system of the subgroup $U_{2}$ of $K$. GAP produces a presentation of $U_{2}$ with 11 generators and 15 relations. Then a presentation of the fundamental group of $M_{2}$ is obtained by adding the relator $f_{1}$ (that is, the relation $f_{1}=1$ ) to this presentation. Using Tietze transformations, GAP simplifies this presentation to the trivial presentation, so $M_{2}$ has trivial fundamental group. The normalizer of $h$ in $\mathbb{A}_{5}$ contains involutions different from $h$; these project to involutions of $M_{2}$ with nonempty fixed point set. By the orbifold geometrization theorem, $M_{2}$ is the 3 -sphere, so $M$ is the 2-fold branched covering of a knot $K_{2}$ in $S^{3}$.

Starting the construction with the element $h=\phi\left(f_{2}\right)$ of order three in $\mathbb{A}_{5}$, one shows in a completely analogous way that $M$ is also the 3 -fold cyclic branched covering of a knot $K_{3}$ in $S^{3}$. We note that $M$ is also the 5 -fold cyclic branched covering of a knot $K_{5}$ in a homology 3 -sphere $M_{5}$, which, however, has nontrivial fundamental group. At present, we do not have projections of the knots $K_{2}$ and $K_{3}$.

Finally, we compute the volume of the hyperbolic homology 3 -sphere $M$. The orbifold $\mathcal{K}$ is obtained by $(5,5)$-surgery on the cusp of the orbifold $\widetilde{\mathcal{P}}$, and $\mathcal{K}$ is a 2 -fold branched covering of the orbifold $\mathcal{P}(5,10)$ shown in Figure $2 \mathrm{~d}$, obtained by $(5,10)$-surgery on the cusp of the Picard orbifold $\mathcal{P}$ (see [MVZ]; see also [DM] for the notion of surgery on orbifolds). The complement of the Borromean rings $\mathcal{B}$ in $S^{3}$ is a regular 24-fold covering, with covering group $\mathbb{S}_{4}$, of the Picard orbifold (see e.g. [MVZ]). By [MVZ, Theorem 2.1 and Remark (A)], the hyperbolic orbifold $\mathcal{K}=\mathcal{P}(5,10)$ has the hyperbolic cone manifold $\mathcal{B}(15 / 2,5 / 4)$, obtained by $(15 / 2,5 / 4)$-surgery on each component of the Borromean rings, as a regular $\mathbb{S}_{4}$-covering. The volume of such a cone manifold can be computed by Weeks' program SnapPea, and one finds the approximate volume $v=6.403214$ for $\mathcal{B}(15 / 2,5 / 4)$. Then $\mathcal{K}$ has volume $2 v / 24$, and the regular $\mathbb{A}_{5}$-covering $M$ of $\mathcal{K}$ has volume $120 v / 24=32.01607$.

This finishes the proof of Theorem 2. There remains the following

Problem. Find regular projections of the dodecahedral link $L$, and of the knots $K_{2}$ and $K_{3}$.

4. $\operatorname{PSL}(2, q)$-actions on $\mathbb{Z}_{2}$-homology 3-spheres. By [MZ, Thm. 1], the only finite simple groups which possibly admit actions on $\mathbb{Z}_{2}$-homology 3 -spheres are the linear fractional groups $\operatorname{PSL}(2, q)$, for odd prime powers $q$. In the following, we show how to construct such actions, for various small values of $q$. 


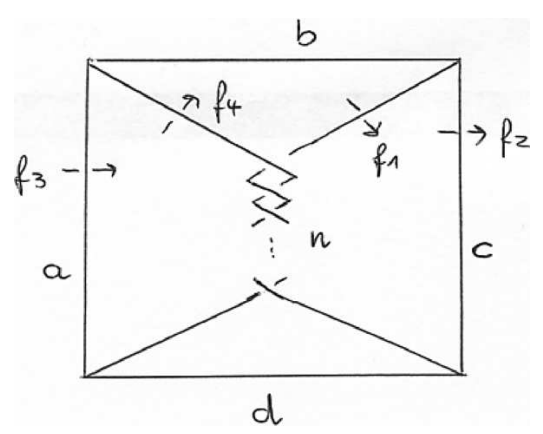

Fig. 3

We consider the orbifold $\mathcal{O}(a, b, c, d ; n)$ whose space is the 3 -sphere and whose singular set is shown in Figure 3 (where $a, b, c$ and $d$ denote branching orders and $n$ the number of half twists between the diagonal edges). Using the Wirtinger algorithm as in Section 2, one obtains the following presentation of the orbifold fundamental group of $\mathcal{O}(a, b, c, d ; n)$ (in the meridian generators indicated in Figure 3):

$$
\left\langle f_{1}, f_{2}, f_{3}, f_{4} \mid f_{1}^{2}, f_{2}^{c}, f_{3}^{b}, f_{4}^{2},\left(f_{1} f_{2}\right)^{b}, f_{3} f_{4} f_{1} f_{2},\left(\left(f_{4} f_{1}\right)^{n} f_{1} f_{2}\right)^{d}\right\rangle .
$$

Let $F_{1}$ denote the orbifold fundamental group of $\mathcal{O}(3,3,3,2 ; 2)$. It follows from the orbifold geometrization theorem that $\mathcal{O}(3,3,3,2 ; 2)$ is hyperbolic (by the lists in $[\mathrm{D}]$ it does not belong to any of the other 3-dimensional geometries). By the Low Index Subgroup Program of GAP we compute the subgroups up to index 30 of $F_{1}$, their normal cores (the largest normal subgroups contained in them), the abelianizations of the cores and their quotient groups. Among other things, we find the following finite quotients $G$ and abelianizations of the corresponding cores (a sequence of integers stands for the product of cyclic groups of these orders). The cores are torsionfree and define hyperbolic 3 -manifolds with isometric $G$-actions; note that, in the cases listed below, these 3 -manifolds are $\mathbb{Z}_{2}$-homology 3 -spheres (equivalently, the abelianizations of their fundamental groups are finite of odd order).

$$
\begin{array}{ll}
\operatorname{PSL}(2,7)(\text { order 168): } & {[7,7,7]=:\left[7^{3}\right] ;} \\
\operatorname{PSL}(2,11)(\text { order } 660): & {\left[11^{3}\right] ;} \\
\operatorname{PSL}(2,13)(\text { order 1092): } & {\left[13^{3}\right] ;} \\
\operatorname{PSL}(2,17)(\text { order 2448): } & {\left[3^{16}, 17^{3}\right] ;} \\
\operatorname{PSL}(2,23)(\text { order 6072): } & {\left[5^{23}, 23^{3}\right] .}
\end{array}
$$

Now let $F_{2}$ denote the fundamental group of the tetrahedral orbifold $\mathcal{O}(3,5,3,2 ; 1)$ (its singular set is the 1-skeleton of a hyperbolic tetrahedron, so this orbifold is also hyperbolic). By applying GAP as above we obtain 
the following quotients $G$ and abelianizations of the corresponding cores:

$$
\begin{array}{ll}
\operatorname{PSL}(2,9)(\text { order } 360): & {[3,3,3,3,3,3]=\left[3^{6}\right] ;} \\
\operatorname{PSL}(2,19)(\text { order } 3420): & {\left[3^{19}, 19^{3}\right] ;} \\
\operatorname{PSL}(2,29)(\text { order 12180): } & {\left[3^{28}, 5^{5}, 29^{3}\right] .}
\end{array}
$$

Using other orbifolds of type $\mathcal{O}(a, b, c, d ; n)$ one easily finds more examples of $\operatorname{PSL}(2, q)$-actions on $\mathbb{Z}_{2}$-homology 3 -spheres. We close with the

Conjecture. (i) For all odd prime powers $q$, the linear fractional group $\operatorname{PSL}(2, q)$ acts on some $\mathbb{Z}_{2}$-homology 3 -sphere.

(ii) All finite groups of odd order act on some $\mathbb{Z}_{2}$-homology 3 -sphere.

\section{Other examples of hyperbolic homology 3 -spheres with iso-} metric $\mathbb{A}_{5}$-actions. Considering the orbifolds $\mathcal{O}(a, b, c, d ; n)$ in Section 4 and Figure 3, and surjections of their fundamental groups onto $\mathbb{A}_{5}$, we find the following examples of hyperbolic homology 3 -spheres with isometric $\mathbb{A}_{5}$ actions.

5.1. Let $F$ denote the orbifold fundamental group $\pi_{1} \mathcal{O}(5,3,3,2 ; 3)$ of the hyperbolic 3 -orbifold $\mathcal{O}(5,3,3,2 ; 3)$ (see Section 4$)$; then $F$ admits a surjection onto $\mathbb{A}_{5}$, and the kernel defines a hyperbolic 3 -manifold $M$ with isometric $\mathbb{A}_{5}$-action. Using GAP as in Sections 3 and 4 , we have checked that $M$ is a homology 3 -sphere, and that $M$ is the 3 -fold and 5 -fold cyclic branched covering of knots in the 3 -sphere. $M$ is also the 2 -fold branched covering of a knot in a homology 3 -sphere which seems to have nontrivial fundamental group (we do not have a proof of this at the moment). In any case, the covering groups of the 3 -fold and 5 -fold cyclic branched coverings generate the isometric $\mathbb{A}_{5}$-action, and no conjugates of them generate a solvable subgroup of the isometry group of $M$ (this follows easily from the list in [MZ] of the possible nonsolvable finite groups acting on homology 3 -spheres). So this seems to be the first example of a hyperbolic 3-manifold which is the 3 -fold and 5 -fold cyclic branched covering of knots in the 3 sphere which are not related in a solvable way (see also [RZ1]).

5.2. Now let $F=\pi_{1} \mathcal{O}(2,5,3,3 ; 9)$. Again $F$ admits a surjection onto $\mathbb{A}_{5}$ whose kernel is a hyperbolic homology 3 -sphere $M$ with isometric $\mathbb{A}_{5}$-action; now $M$ is the 2-fold and 3 -fold cyclic branched covering of knots in the 3 -sphere (and the 5-fold cyclic branched covering of a knot in a homology 3sphere), and these two knots are related by a standard dihedral construction. We note that the manifold $M$ is a maximally symmetric $\mathbb{A}_{5}$-manifold in the sense of [Z2]: it admits a Heegaard splitting of genus $g=6$ invariant under the $\mathbb{A}_{5}$-action, where $\mathbb{A}_{5}$ realizes the maximal order $12(g-1)$ of a finite group of orientation-preserving diffeomorphisms of a handlebody of genus 6 . 
5.3. Other examples of hyperbolic homology 3 -spheres with $\mathbb{A}_{5}$-actions can be obtained by the orbifolds $\mathcal{O}(2,3,3,3 ; 5), \mathcal{O}(2,3,3,3 ; 21)$ and $\mathcal{O}(3,3,3,2 ; 4)$. Again the first two manifolds are maximally symmetric $\mathbb{A}_{5^{-}}$ manifolds.

5.4. Let $\mathcal{K}(3,3,3,2 ; n)$ denote the series of 3 -orbifolds in Figure 4 parametrized by the number of half-twists $n$ which simultaneously generalizes the orbifolds $\mathcal{O}(3,3,3,2 ; n)$ and the Kuratowski orbifold $\mathcal{K}$ of Section 3. Our impression (checked for many small values of $n$ ) is that for all $n \geq 0$ there is a surjection of the orbifold fundamental group onto $\mathbb{A}_{5}$ whose kernel has trivial abelianization and hence defines a homology 3 -sphere with $\mathbb{A}_{5}$-action (for $n=0$ one gets a homology 3 -sphere which is the $\mathbb{A}_{5}$-equivariant connected sum of two copies of the Poincaré sphere, by identifying the boundaries of regular neighbourhoods of the unique global fixed points of the two $\mathbb{A}_{5^{-}}$ actions).

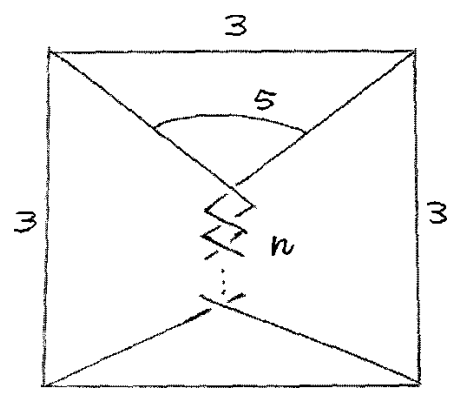

Fig. 4

5.5. Let $p$ be an odd prime. Except for the ubiquitous $\mathbb{A}_{5}$ we have not found a single example of a nonabelian simple group $G$ acting on a 3 -manifold $M$ which is a $p$-fold cyclic branched covering of a knot or link in the 3 -sphere whose covering group is contained in $G$. The closest we found in this direction is the following. The fundamental group of the hyperbolic tetrahedral orbifold $\mathcal{O}(3,5,3,2 ; 1)$ admits a surjection onto the alternating or linear fractional group $\mathbb{A}_{6} \cong \operatorname{PSL}(2,9)$ of order 360 such that the kernel has abelianization $\left(\mathbb{Z}_{3}\right)^{6}$ and hence defines a hyperbolic $\mathbb{Z}_{2}$-homology 3 -sphere $M$ with an isometric $\mathbb{A}_{6}$-action. The Sylow 3-subgroup of $\mathbb{A}_{6}$ is isomorphic to $\mathbb{Z}_{3} \times \mathbb{Z}_{3}$, and we have checked as in Section 3 (i.e. using the orbifold geometrization theorem) that the quotient $M / \mathbb{Z}_{3} \times \mathbb{Z}_{3}$ is the 3 -sphere, so $M$ is a regular $\mathbb{Z}_{3} \times \mathbb{Z}_{3}$-covering of a link in the 3 -sphere (note that the involution corresponding to the lower edge of the tetrahedral orbifold $\mathcal{O}(3,5,3,2 ; 1)$ normalizes the two elements of order 3 corresponding to the two vertical edges). It would be interesting to have an example of this kind with a cyclic covering group but we believe that such an example does not exist if $G$ is different from $\mathbb{A}_{5}$. 


\section{References}

[A] M. A. Armstrong, The fundamental group of the orbit space of a discontinuous group, Proc. Cambridge Philos. Soc. 64 (1968), 299-301.

[BP] M. Boileau and J. Porti, Geometrization of 3-orbifolds of cyclic type, Astérisque 272 (2001), 1-208.

[Br] G. Bredon, Introduction to Compact Transformation Groups, Academic Press, New York, 1972.

[CHK] D. Cooper, C. D. Hodgson and S. P. Kerckhoff, Three-dimensional orbifolds and cone-manifolds, Math. Soc. Japan Mem. 5 (2000).

[D] W. Dunbar, Geometric orbifolds, Rev. Mat. Univ. Complut. 1 (1988), 67-99.

[DM] W. Dunbar and R. Meyerhoff, Volumes of hyperbolic 3-orbifolds, Indiana Univ. Math. J. 43 (1994), 611-637.

[F] B. Fine, Algebraic Theory of the Bianchi Groups, Monographs and Textbooks in Pure and Appl. Math. 129, Dekker, New York, 1989.

[G] C. McA. Gordon, Some aspects of classical knot theory, in: Knot Theory, J. C. Hausmann (ed.), Lecture Notes in Math. 685, Springer, 1977, 1-60.

[MZ] M. Mecchia and B. Zimmermann, On finite groups acting on $\mathbb{Z}_{2}$-homology 3spheres, Math. Z. 248 (2004), 675-693.

[MVZ] A. Mednykh, A. Vesnin and B. Zimmermann, Surgery on small volume hyperbolic 3-orbifolds, Sibirsk. Mat. Zh. 42 (2001), 318-331 (in Russian); English transl.: Siberian Math. J. 42 (2001), 271-281.

[N1] M. Nakao, On the $Z_{2} \oplus Z_{2}$ branched coverings of spatial $K_{4}$-graphs, in: Knots 90 (Osaka, 1990), A. Kawauchi (ed.), de Gruyter, Berlin, 1992, 103-116.

[N2] - On the $Z_{2} \oplus Z_{2}$ branched coverings of spatial $\theta$-curves, Kobe J. Math. 9 (1992), 89-99.

[RZ1] M. Reni and B. Zimmermann, Hyperbolic 3-manifolds as cyclic branched coverings, Comment. Math. Helv. 76 (2001), 300-313.

[RZ2] - - - Standard situations for cyclic branched coverings of hyperbolic knots, Math. Z. 239 (2002), 415-424.

[RZ3] -, - Isometry groups of hyperbolic 3-manifolds which are cyclic branched coverings, Geom. Dedicata 74 (1999), 23-35.

[RZ4] -, - Finite simple groups acting on 3-manifolds and homology spheres, Rend. Istit. Mat. Univ. Trieste 32 (2001), suppl. 1, 305-315.

[W] F. Waldhausen, Über Involutionen der 3-Sphäre, Topology 8 (1969), 81-91.

[Z1] B. Zimmermann, On finite simple groups acting on homology 3-spheres, Topology Appl. 125 (2002), 199-202.

[Z2] -, Genus actions of finite groups on 3-manifolds, Michigan Math. J. 43 (1996), 593-610.

Dipartimento di Matematica e Informatica

Università degli Studi di Trieste

34100 Trieste, Italy

E-mail: zimmer@units.it

Received 1 March 2004;

in revised form 4 November 2004 